\title{
BMJ Open Predicting poorer health outcomes in older community-dwelling patients with multimorbidity: prospective cohort study assessing the accuracy of different multimorbidity definitions
}

\author{
Maxime Sasseville, ${ }^{1,2}$ Susan M Smith, ${ }^{3}$ Lisa Freyne ${ }^{3}$ Ronald McDowell, ${ }^{3,4}$ \\ Fiona Boland, ${ }^{3,5}$ Martin Fortin, ${ }^{6}$ Emma Wallace ${ }^{3}$
}

To cite: Sasseville M, Smith SM, Freyne L, et al. Predicting poorer health outcomes in older community-dwelling patients with multimorbidity: prospective cohort study assessing the accuracy of different multimorbidity definitions. BMJ Open 2019;9:e023919. doi:10.1136/ bmjopen-2018-023919

- Prepublication history for this paper is available online. To view these files, please visit the journal online (http://dx.doi. org/10.1136/bmjopen-2018023919).

Received 1 May 2018

Revised 9 0ctober 2018 Accepted 23 November 2018

Check for updates

(c) Author(s) (or their employer(s)) 2019. Re-use permitted under CC BY-NC. No commercial re-use. See rights and permissions. Published by BMJ.

For numbered affiliations see end of article.

Correspondence to Mr Maxime Sasseville; maxime1_sasseville@uqac.ca

\section{ABSTRACT}

Purpose Multimorbidity is commonly defined and measured using condition counts. The UK National Institute for Health Care Excellence Guidelines for Multimorbidity suggest that a medication-orientated approach could be used to identify those in need of a multimorbidity approach to management.

Objectives To compare the accuracy of medication-based and diagnosis-based multimorbidity measures at higher cut-points to identify older community-dwelling patients who are at risk of poorer health outcomes.

Design A secondary analysis of a prospective cohort study with a 2-year follow-up (2010-2012).

Setting 15 general practices in Ireland.

Participants 904 older community-dwelling patients. Exposure Baseline multimorbidity measurements based on both medication classes count (MCC) and chronic disease count (CDC).

Outcomes Mortality, self-reported health related quality of life, mental health and physical functioning at follow-up.

Analysis Sensitivity, specificity, positive predictive values (PPV) and negative predictive values (NPV) adjusting for clustering by practice for each outcome using both definitions.

Results of the 904 baseline participants, 53 died during follow-up and 673 patients completed the follow-up questionnaire. At baseline, 223 patients had 3 or more chronic conditions and 89 patients were prescribed 10 or more medication classes. Sensitivity was low for both MCC and CDC measures for all outcomes. For specificity, MCC was better for all outcomes with estimates varying from $88.8 \%$ (95\% Cl 85.2\% to 91.6\%) for physical functioning to $90.9 \%$ (95\% Cl $86.2 \%$ to $94.1 \%$ ) for self-reported health-related quality of life. There were no differences between MCC and CDC in terms of PPV and NPV for any outcomes.

Conclusions Neither measure demonstrated high sensitivity. However, MCC using a definition of 10 or more regular medication classes to define multimorbidity had higher specificity for predicting poorer health outcomes. While having limitations, this definition could be used for proactive identification of patients who may benefit from targeted clinical care.

\section{Strengths and limitations of this study}

- This study compares use of medication classes count with the more traditional method of chronic disease counts to define multimorbidity. This approach could be used proactively in the clinical setting to identify higher risk people.

- Our study used a large dataset with robust data collection from electronic health records combined with linked national pharmacy claims data and a patient questionnaire for self-reported outcomes.

- This study only included older patients with multimorbidity, further research would be needed to validate these results in other populations.

- This study is a secondary analysis and as such is limited to the data collected from the recruited population in the original cohort study.

\section{INTRODUCTION}

A high proportion of patients consulting in primary healthcare present with multimorbidity, defined generally as the presence of at least two chronic medical conditions. ${ }^{1}$ Multimorbidity has a significant impact across the age ranges but is more common in older patients and is associated with poorer quality of life,${ }^{23}$ psychological distress, ${ }^{4-6}$ loss of physical function, ${ }^{7}$ polypharmacy and adverse drugs events ${ }^{8}$ and care duplication and inconsistencies. ${ }^{9} 10$ Within the broad multimorbidity population, outcomes are poorer in patients with more complex multimorbidity, which has been defined previously in terms of higher numbers of conditions or higher healthcare utilisation.

Even though associations with poorer health outcomes are clear, identifying older patients with multimorbidity who will benefit from a community-based intervention is difficult due to the heterogeneity of multimorbidity 
definitions and measures, used in both public health and clinical interventions. ${ }^{11}$ Existing trials have based inclusion on the number of conditions along with other markers of risk such as older age or high healthcare utilisation. ${ }^{12}$ The UK National Institute for Health and Care Excellence (NICE) 2016 Guidance on Multimorbidity recommends that health practitioners should proactively identify patients that could benefit from a multimorbidity approach to clinical care. The NICE Guidance suggests considering a multimorbidity approach to care for adults of any age who are prescribed $\geq 10$ medications with the advantage that this information can be retrieved from the electronic health record. ${ }^{13}$ This approach to care is patient-centred as it follows patient goals and preference of care, focusing on quality of life by reducing treatment burden, adverse events, and unplanned care and improving services coordination. Polypharmacy is a marker of multimorbidity and patients identified this way can be regarded as having multimorbidity and offered broad interventions beyond medicines management. For condition count multimorbidity measurement, the literature suggests using $\geq 3$ chronic conditions to identify patients with higher needs. ${ }^{\text {st } 115}$

This study aimed to examine the accuracy of medication-based versus condition count-based definitions of multimorbidity in predicting poorer health outcomes for older community-dwelling patients.

\section{METHODS}

The Strengthening The Reporting of Observational Studies in Epidemiology guidelines were used to guide the conduct and reporting of this study. ${ }^{16}$

\section{Study design and population}

This is a secondary analysis of a 2-year, prospective cohort study that was established to examine potentially inappropriate prescribing and adverse health outcomes in older community-dwelling patients. ${ }^{17}$ The study population was recruited from 15 randomly selected general practices in Leinster, Ireland for a 2-year (2010-2012) prospective cohort study involving older community-dwelling patients. Proportionate stratified sampling was carried out based on the overall required sample size and the total number of eligible patients per practice assuming a $50 \%$ response rate and of 3070 eligible patients, a total of 1764 were invited to participate. ${ }^{1718}$ Of this group, 152 were ineligible on invitation based on eligibility criteria and 125 were not contactable resulting in 1487 patients eligible for participation.

\section{Study inclusion criteria}

1. Age $\geq 70$ years on 1 January 2010.

2. in receipt of a valid general medical services card, which is means tested and entitles the holder to free public medical services including general practitioner (GP) care.
Exclusion criteria

1. Receiving palliative care.

2. Cognitive impairment at the level that would affect their ability to complete the outcome measure (defined as Mini Mental State Examination $\leq 20$ ).

3. Significant hearing/speech/visual impairment.

4. Currently experiencing a psychotic episode.

5. Hospitalised long-term, in a nursing home, homeless or in sheltered accommodation.

6. Recent bereavement (within 4 weeks).

Of 1487 eligible and invited to participate at baseline (T0) a total of $904(61 \%)$ agreed. ${ }^{17} 18$ Two study populations are presented depending on the outcome of interest:

a. Patients who either completed a 2-year follow-up (T1) or died before $\mathrm{T} 1$ for mortality analysis $(\mathrm{n}=726)$.

b. Patients who completed T1 self-reported questionnaires for patient reported health outcomes analysis $(\mathrm{n}=673)$.

\section{Demographic data collected}

Sociodemographic variables collected included age, deprivation, gender, social class, education, marital status and living arrangements. Age and gender were collected from the GP electronic medical record. The deprivation score was obtained with the geocoded patients address based on the Small Area Health Research Unit which uses electoral division. ${ }^{19}$ Education levels were classified as basic education (no formal education, primary education or lower secondary education only) or upper and postsecondary (all other higher levels of education). Social class was classified as unskilled (unskilled, gainfully occupied, unknown) or skilled (all other categories). Marital status was classified as married, separated/ divorced, widowed and single/never married. Living arrangements were classified as living with husband/ wife/life partner, family/relatives, living alone and other.

\section{Exposure of interest: multimorbidity measures}

Two measures of multimorbidity were selected based on current guidelines and literature. $^{1415}$

\section{Medication classes count (MCC)}

The number of regular prescribed medication classes was calculated by linkage to the national Health Services Executive-Primary Care Reimbursement Scheme pharmacy claims database. The number of medication classes prescribed to the patient were classified using the first three characters of the WHO-ATC classification system and the sample was divided using a cut-off definition of $\geq 10$ prescribed medication classes. ${ }^{20}$ The NICE guidelines indicate that a patient with $\geq 10$ prescribed medicines and an additional risk factor would benefit from a multimorbidity approach. ${ }^{21}$ As all cohort participants were $\geq 70$ years, this population was considered as having an additional risk factor. 
Chronic disease count (CDC)

At baseline, chronic diseases were collected from the GP electronic medical record by eight trained medical students using standardised data collection forms. A disease count proposed by Barnett et al which includes 40 chronic diseases on the basis of disease prevalence and severity was used to define multimorbidity. ${ }^{22} \mathrm{~A}$ cut-off of $\geq 3$ chronic diseases was used to identify multimorbidity. ${ }^{1415}$

\section{Primary outcomes}

Mortality and patient reported outcome measures (PROM) were selected to identify patients with poorer health at follow-up. The study examined poorer self-reported health between T0 (baseline) and T1 (2 years). The PROMs were dichotomised, as described below to identify changes in outcome over the 2 years.

\section{Mortality}

Mortality was assessed by examining each participant's GP electronic medical record. Where there was any query regarding the date of death, it was double checked using a national repository of deaths in Ireland.

Health-related quality of life

The Euro-Qual 5 Dimensions (EQ-5D) is a generic instrument widely used to assess health-related quality of life by using ordinal scaling to assess fives domains: mobility, self-care, usual activities, pain/discomfort and anxiety/ depression. Poorer self-reported health was defined using the Paretian principles method described by Devlin et $a l^{23}$ Following these principles, poorer health-related quality of life was operationalised as a decline in a greater number of domains scores compared with stable or improved domains scores. ${ }^{23}$

\section{Mental health}

The Hospital Anxiety and Depression Scale (HADS) is a measure assessing levels of anxiety and depression independently, which are then classified as normal, mild, moderate or severe. ${ }^{24}$ Poorer self-reported mental health was defined as a higher score at follow up-compared with baseline according to the HADS. ${ }^{24}$

\section{Physical functioning}

The Vulnerable Elders Survey (VES-13) is a patient-reported outcome measure used to identify older patients at risk of functional decline. ${ }^{25}$ In the derivation study for this tool, patients who scored $\geq 3$ had four times the risk of death or functional decline over a 2-year period than patients who scored $<3 .^{25}$ Accordingly, patients were classified as having poorer self-reported health if study participants who scored $1<3$ at baseline (T0) scored $\geq 3$ at follow-up (T1).

\section{Statistical analysis}

Descriptive statistics are presented to describe patient characteristics. For categorical measures, the number of patients and percentage were calculated, and for continuous measures, the mean and SD. For continuous scales which showed evidence of, or were expected to show some skew, a median and interquartile range were presented. A $X^{2}$ test, t-test or Mann-Whitney test was used as appropriate, adjusting for clustering by practice, to examine possible associations between patient characteristics and multimorbidity measures (patients with $\geq 10 \mathrm{vs}$ $<10$ prescribed medication classes and patients with $\geq 3 \mathrm{vs}$ $<3$ chronic diseases).

The sensitivity, specificity, positive predictive values (PPV) and negative predictive values (NPV), along with 95\% CIs, were calculated adjusting for clustering by practice using Stata V.15 (StataCorp. 2017) for each outcome and using both exposures of interest (MCC and CDC). For our analyses, sensitivity is the probability of a patient who experiences the health outcome of interest (ie, death or decline in PROM at follow-up) having multimorbidity at baseline, specificity is the probability of a patient who does not experience the health outcome at follow-up not having multimorbidity at baseline, the PPV is the probability of a patient with multimorbidity at baseline having the health outcome at follow-up and the NPV is the probability of a patient without multimorbidity at baseline not having the health outcome at follow-up.

\section{Risk of bias}

The risk of bias was assessed in the cohort study using The Cochrane risk of bias tool for non-randomised studies. ${ }^{26}$

\section{Patient and public involvement}

This study is a secondary analysis of a cohort study which started enrolment in 2010. No patients or members of the public were involved in its design phase.

\section{RESULTS \\ Participants}

A total of 223 participants $(30.7 \%)$ met the criteria for the CDC definition for multimorbidity, and 89 patients (12.3\%) met the criteria for the MCC definition. Overall, 61 patients met both criteria. The median age of the total sample was 76.4 years, a total of $348(47.9 \%)$ participants were men, $565(77.8 \%)$ were classified as coming from the skilled social class and $433(59.6 \%)$ reported a basic level of education. Descriptive characteristics of the patients identified by the CDC and the MCC cut-offs are presented in table 1. Patients with a disease count $\geq 3$, compared with those with a disease count $<3$, were on average older and reported less formal education. Patients prescribed $\geq 10$ medications classes were on average older, women and had less formal education compared with patients prescribed $<10$ medication classes.

\section{OUTCOMES}

The proportion of patients defined as having multimorbidity using both definitions who died or reported poorer health outcomes are presented in table 2. Patients with 


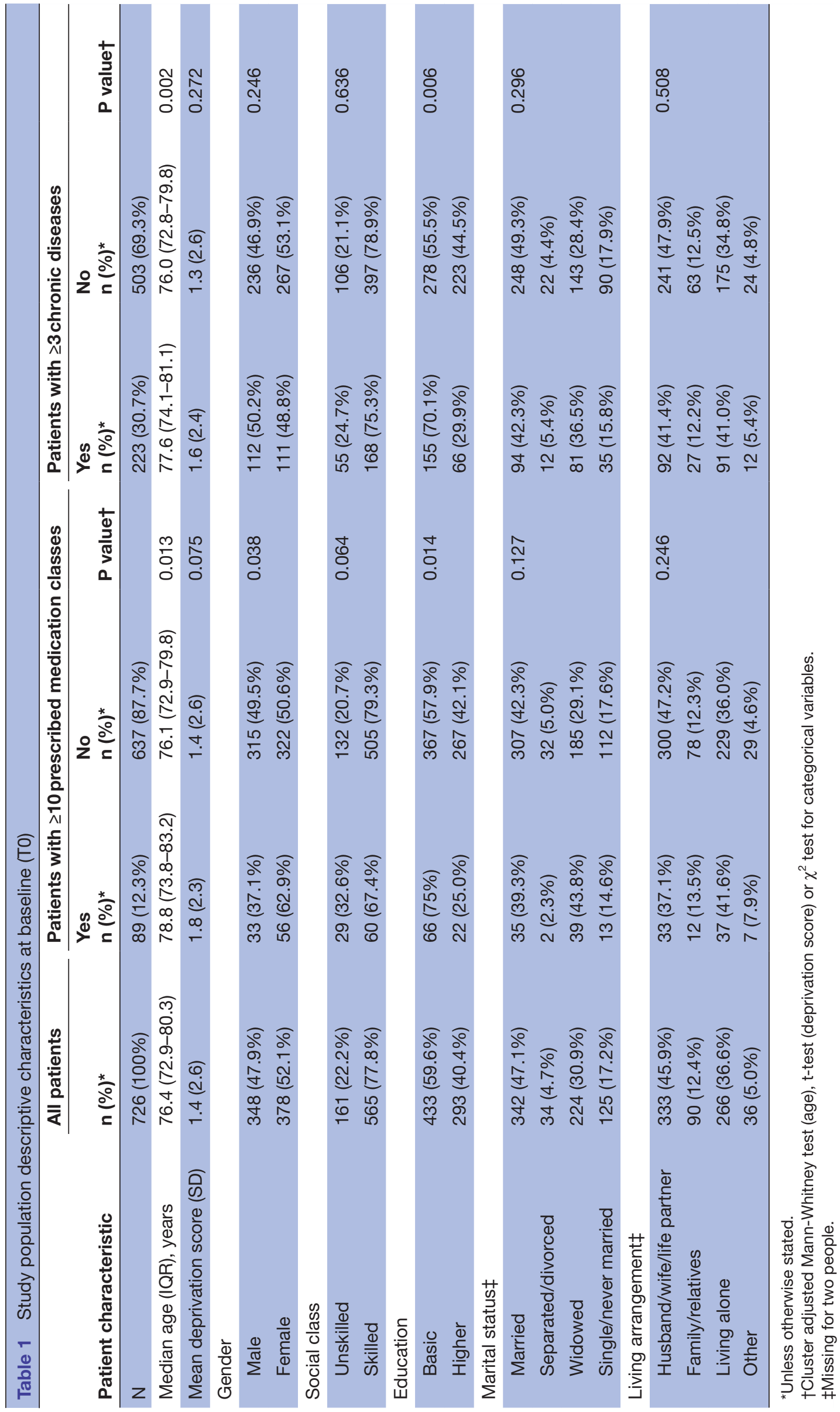


Table 2 Patients with multimorbidity according to MCC or CDC and outcomes of death, decline in health-related quality of life, decline in physical functioning and decline in psychological well-being

\begin{tabular}{|c|c|c|c|c|c|c|}
\hline \multirow[b]{2}{*}{ Outcome } & \multicolumn{3}{|l|}{ MCC } & \multicolumn{3}{|l|}{ CDC } \\
\hline & $\begin{array}{l}\text { Patients with }<10 \\
\text { medications }\end{array}$ & $\begin{array}{l}\text { Patients with } \geq 10 \\
\text { medications }\end{array}$ & $\begin{array}{l}\text { Cluster adjusted } \\
\chi^{2} p \text { value }\end{array}$ & $\begin{array}{l}\text { Patients with } \\
<3 \text { chronic } \\
\text { diseases }\end{array}$ & $\begin{array}{l}\text { Patients with } \\
\geq 3 \text { chronic } \\
\text { diseases }\end{array}$ & $\begin{array}{l}\text { Cluster adjusted } \\
\mathbf{X}^{2} \mathbf{p} \text { value }\end{array}$ \\
\hline \multicolumn{7}{|c|}{ Death $(n=724)$} \\
\hline No & $598(94.0 \%)$ & $73(83.0 \%)$ & $<0.001$ & $472(94.0 \%)$ & $199(89.6 \%)$ & 0.054 \\
\hline Yes & $38(6.0 \%)$ & $15(17.0 \%)$ & & $30(6.0 \%)$ & $23(10.4 \%)$ & \\
\hline No & $393(68.3 \%)$ & $39(63.9 \%)$ & 0.443 & $310(68.0 \%)$ & $122(67.8 \%)$ & 0.820 \\
\hline Yes & $182(31.7 \%)$ & $22(36.0 \%)$ & & $146(32.0 \%)$ & $58(32.2 \% \%)$ & \\
\hline \multicolumn{7}{|c|}{ Decline in physical functioning (VES-13) $(n=673)$} \\
\hline No & $525(87.7 \%)$ & $66(89.2 \%)$ & 0.622 & $418(88.4 \%)$ & $173(86.5 \%)$ & 0.768 \\
\hline Yes & $74(12.4 \%)$ & $8(10.8 \%)$ & & $55(11.6 \%)$ & $27(13.5 \%)$ & \\
\hline \multicolumn{7}{|c|}{ Decline in psychological well-being (HADS) $(n=649)$} \\
\hline
\end{tabular}

CDC, chronic disease count; EQ5D, Euro-Qual 5 Dimensions; HADS, Hospital Anxiety and Depression Scale; MCC, medication classes count; VES-13, Vulnerable Elders Survey.

$\geq 10$ medication classes had a significantly higher mortality rate compared with patients with $<10$ medication classes ( $17 \%$ vs $6 \%, \mathrm{p}<0.001)$. There was no difference in the other outcomes measured. Patients with a disease count of $\geq 3$, compared with those with a disease count of $<3$, had a significantly higher decline in psychological wellbeing $(19.5 \%$ vs $11.7 \%, \mathrm{p}=0.017)$ but there was no difference in any of the other outcomes.

The sensitivity, specificity, PPV and NPV along with 95\% CIs adjusted for clustering by practice are presented in table 3 for both definitions of multimorbidity for each outcome of interest. For mortality, specificity was higher for MCC $(89.0 \%$; $95 \%$ CI $86.0 \%$ to $91.5 \%$ ) compared with the CDC (70.3\%; 95\% CI $63.5 \%$ to $76.3 \%)$. For decline in health-related quality of life, both CDC and MCC measures had low sensitivity, however, the CDC (28.5\%; $95 \%$ CI $22.1 \%$ to $35.9 \%$ ) was higher than the MCC $(10.5 \%$; $95 \%$ CI $7.6 \%$ to $14.4 \%)$. In terms of specificity, MCC (90.9\%; $95 \%$ CI $86.2 \%$ to $94.1 \%)$ was higher when compared with the CDC measure $(71.9 \%$; $95 \%$ CI $64.8 \%$ to $78.1 \%$ ).

Similar patterns were reported for decline in physical functioning and psychological functioning as were seen for decline in health-related quality of life. Specificity was moderate for the CDC measure for both decline in physical functioning $(71.9 \%$; $95 \%$ CI $64.8 \%$ to $78.1 \%$ ) and decline in psychological functioning $(71.1 \%$; $95 \% \mathrm{CI}$ $64.6 \%$ to $76.9 \%$ ). The MCC demonstrated higher specificity (decline in physical functioning: $88.8 \%$; $95 \% \mathrm{CI}$ $85.2 \%$ to $91.6 \%$ and decline in psychological functioning: $89.9 \%$; $95 \%$ CI $86.2 \%$ to $92.7 \%$ ).

\section{Risk of bias}

The risk of bias is reported fully in the primary cohort study previously. ${ }^{17}$ Overall, the risk of bias was low regarding losses to follow-up. As this is a secondary analysis, exclusion of participants with cognitive impairment and the small sample of participants with $\geq 15$ medication classes could reduce generalisability. ${ }^{26}$

\section{DISCUSSION}

\section{Main results}

In this population of community-dwelling older patients, sensitivity was low for both the MCC and CDC measures for all outcomes. However, for self-reported health-related quality of life, psychological well-being and physical functioning at follow-up, the CDC measure was more sensitive. There was no difference in sensitivity between measures for the outcome of death. In terms of specificity, the MCC approach was better for all outcomes with estimates varying from $88.8 \%$ (95\% CI $85.2 \%$ to $91.6 \%$ ) for physical functioning to $90.9 \%$ (95\% CI $86.2 \%$ to $94.1 \%$ ) for self-reported health-related quality of life.

With high specificity, the MCC definition has better potential to proactively 'rule-in' patients with multimorbidity who are more likely to have poorer health outcomes compared with the more widely used condition count approach. There are also fewer patients identified using the MCC definition, which is more manageable from a clinical or organisational perspective. However, both definitions had insufficient sensitivity, showing a limited potential to accurately 'rule out' patient at risk of poorer health outcomes in this population. Ideally a definition with both high sensitivity and specificity could be used to target multimorbidity interventions but existing risk stratification models have similar limitations. ${ }^{27}$ 


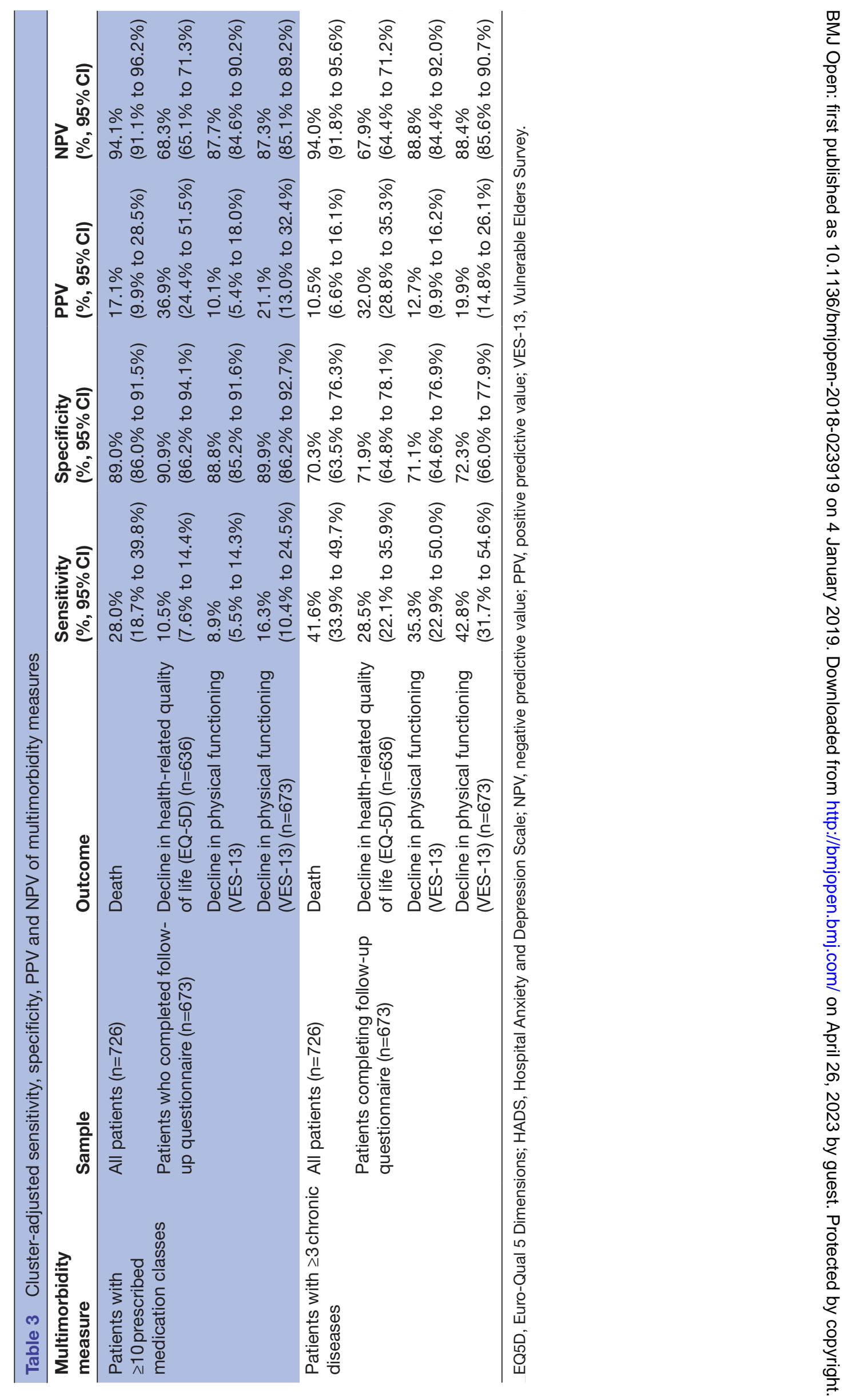




\section{Strengths and limitations}

In this cohort study, the MCC variable was obtained from linked national pharmacy claims data and the number of chronic diseases count was obtained via review of the participants' electronic medical record, which adds to the robustness to the data used in this analysis. A main strength of the study is that the dataset includes a variety of outcomes including mortality and patient-reported outcomes.

The first limitation of this study is inherent to the secondary analysis design. By using previously collected data, it is not possible to align data collection directly with the goals of the current study, however we did have a wide range of patient reported and chart data available for analysis. The cohort study sample was limited to a community-dwelling older people without cognitive, visual or hearing impairment. However, recent studies indicate that multimorbidity is not just a feature of ageing but is also prevalent in younger populations. ${ }^{128}{ }^{29}$ We limited the number of chronic conditions identified in the records to 40 prespecified conditions. Another potential limitation is the collection of data from medical records as there may have been some variation in recording of conditions. Medication classes were used as the predictor of interest as per the WHO-ATC classification system rather than individual medications. Further research is needed to validate our findings in larger samples

\section{Comparison with existing literature}

Previous studies have reported mixed results concerning the predictive power of multimorbidity definitions for different outcomes. Several studies have showed that a weighted diagnosis count, the Charlson index was an suitable measure to predict mortality ${ }^{30-32}$ However, a large cohort study ( $\mathrm{n}=95$ 372) comparing six measure of multimorbidity reported that the number of prescribed medications was the most accurate multimorbidity measurement to predict future GP and practice nurses consultations and that it was also the second most accurate measure to predict mortality, just behind the Charlson index. ${ }^{33}$ A previous analysis of the current cohort study data compared five continuous count-based definitions of multimorbidity and reported poor discrimination in predicting hospital admissions and self-reported functional decline for all multimorbidity measures, with the medication class-based definition performing marginally better than diagnosis-based definitions. ${ }^{34}$ A previous study reported that using a $\geq 10$ medication class cut-off to measure multimorbidity performed similarly to another risk score with low sensitivity and high specificity, when applied in clinical settings to predict cardiovascular disease risk. ${ }^{27}$ Our findings are build on this previous research, by comparing the predictive power of a medication class count definition against a disease count definition using pragmatic cut-off points and also examining a range of self-reported health outcomes in addition to mortality. Research to date has highlighted the limitations of multimorbidity measures in predicting adverse events and work in this area is now expanding to include biomarkers in an effort to address these limitations. ${ }^{35} 36$

\section{Implications for future research and clinical practice}

Further research should assess the accuracy of multimorbidity measures in a larger range of primary care populations, including middle-aged patients with multimorbidity and older patients with cognitive decline. Larger sample sizes are needed to test the $\geq 15$ medications cut-off measure for multimorbidity also recommended as an alternative in the UK NICE Multimorbidity Guidance. ${ }^{21}$ In our study, there was little difference in medication and condition count measures in identifying older people at higher risk of poor health outcomes but MCC demonstrated higher specificity showing a slight difference in age, suggesting that the medication definition might better identify older people at higher risk. While it shares some limitations with other multimorbidity measures, clinicians and researchers can follow the expert consensus in the UK NICE Multimorbidity Guidance recommendations by using MCC to identify higher risk patients with multimorbidity as it is easy to use and offers a pragmatic approach and potential for identification of patients through prescribing or electronic health records.

\section{CONCLUSIONS}

This study shows that using two measures of multimorbidity, a MCC cut-off of $\geq 10$ and a CDC of $\geq 3$ chronic diseases had low sensitivity in relation to predicting mortality, self-reported health-related quality of life, mental health and physical functioning, although the CDC was slightly more sensitive for the majority of outcomes. The MCC approach demonstrated higher specificity for mortality and decline in health status, making it possible to rule-in a small sample of patients identified with a risk of poorer health outcomes with a low rate of false positives. However, the low sensitivity means that some of those identified as low risk may also experience poorer health outcomes.

\section{Author affiliations}

${ }^{1}$ Health Sciences, Université du Québec à Chicoutimi, Chicoutimi, Quebec, Canada ${ }^{2}$ Health Science Research, Universite de Sherbrooke, Chicoutimi, Quebec, Canada ${ }^{3}$ Department of General Practice, HRB Centre for Primary Care Research, Royal College of Surgeons in Ireland (RCSI), Dublin, Ireland

${ }^{4}$ Cancer Epidemiology and Health Services Research Group, Centre for Public Health, School of Medicine, Dentistry and Biomedical Sciences, Queen's University, Belfast, Ireland

${ }^{5}$ Division of Population Health Sciences (PHS), HRB Centre For Primary Care Research , Royal College of Surgeons in Ireland (RCSI), Dublin, Ireland

${ }^{6}$ Family Medicine, Université de Sherbrooke, Chicoutimi, Quebec, Canada

Acknowledgements The authors thank Dr Caitriona Cahir who led the collection of baseline data for the cohort.

Contributors MS, SMS and EW conceptualised the study, MS and FB analysed the data and SS, EW, FB and RM took part in the interpretation of results. MS wrote the original draft and SS, EW, FB, RM, MF and LF contributed to the editing and reviewing of the paper. 
Funding The original cohort study was supported by the Health Research Board of Ireland under the Research Training Fellowship for Healthcare Professionals award, grant no. HPF/2012/20 and was conducted as part of the HRB Scholar's programme in Health Services Research (grant no. PhD/2007/16) at the HRB Centre for Primary Care Research, grant HRC/2007/1. Maxime Sasseville was supported by the Ireland Canada University Foundation (ICUF) for the travel and allocation fees to complete this secondary analysis study.

Competing interests None declared.

Patient consent for publication Not required.

Ethics approval Royal College of Surgeons in Ireland (RCSI) Human Research Ethics committee.

Provenance and peer review Not commissioned; externally peer reviewed.

Data sharing statement The database from the cohort study is available from Dr EW by local access only. Please contact corresponding author.

Open access This is an open access article distributed in accordance with the Creative Commons Attribution Non Commercial (CC BY-NC 4.0) license, which permits others to distribute, remix, adapt, build upon this work non-commercially, and license their derivative works on different terms, provided the original work is properly cited, appropriate credit is given, any changes made indicated, and the use is non-commercial. See: http://creativecommons.org/licenses/by-nc/4.0/.

\section{REFERENCES}

1. Fortin M, Bravo G, Hudon C, et al. Prevalence of multimorbidity among adults seen in family practice. Ann Fam Med 2005;3:223-8.

2. Fortin M, Bravo G, Hudon C, et al. Relationship between multimorbidity and health-related quality of life of patients in primary care. Qual Life Res 2006;15:83-91.

3. Brettschneider $\mathrm{C}$, Leicht $\mathrm{H}$, Bickel $\mathrm{H}$, et al. Relative impact of multimorbid chronic conditions on health-related quality of life-results from the MultiCare Cohort Study. PLoS One 2013;8:e66742.

4. Gunn JM, Ayton DR, Densley K, et al. The association between chronic illness, multimorbidity and depressive symptoms in an Australian primary care cohort. Soc Psychiatry Psychiatr Epidemiol 2012;47:175-84.

5. Fortin M, Bravo G, Hudon C, et al. Psychological distress and multimorbidity in primary care. Ann Fam Med 2006;4:417-22.

6. Read JR, Sharpe L, Modini M, et al. Multimorbidity and depression: a systematic review and meta-analysis. J Affect Disord 2017;221:36-46.

7. Bayliss EA, Bayliss MS, Ware JE, et al. Predicting declines in physical function in persons with multiple chronic medical conditions: what we can learn from the medical problem list. Health Qual Life Outcomes 2004;2:47.

8. Guthrie B, McCowan C, Davey P, et al. High risk prescribing in primary care patients particularly vulnerable to adverse drug events: cross sectional population database analysis in Scottish general practice. BMJ 2011;342:d3514.

9. Guthrie B, Payne K, Alderson P, et al. Adapting clinical guidelines to take account of multimorbidity. BMJ 2012;345:e6341.

10. Hughes LD, McMurdo ME, Guthrie B. Guidelines for people not for diseases: the challenges of applying UK clinical guidelines to people with multimorbidity. Age Ageing 2013;42:62-9.

11. Almirall J, Fortin M. The coexistence of terms to describe the presence of multiple concurrent diseases. J Comorb 2013;3:4-9.

12. Smith SM, Wallace E, O'Dowd T, et al. Interventions for improving outcomes in patients with multimorbidity in primary care and community settings. Cochrane Database Syst Rev 2016;9.

13. Farmer C, Fenu E, O'Flynn N, et al. Clinical assessment and management of multimorbidity: summary of NICE guidance. BMJ 2016;354:354.

14. Fortin M, Stewart M, Poitras ME, et al. A systematic review of prevalence studies on multimorbidity: toward a more uniform methodology. Ann Fam Med 2012;10:142-51.
15. Harrison $\mathrm{C}$, Britt $\mathrm{H}$, Miller G, et al. Examining different measures of multimorbidity, using a large prospective cross-sectional study in Australian general practice. BMJ Open 2014;4:e004694.

16. von Elm E, Altman DG, Egger M, et al. The Strengthening the Reporting of Observational Studies in Epidemiology [STROBE] statement: guidelines for reporting observational studies. Gac Sanit 2008;22:144-50.

17. Wallace E, McDowell R, Bennett K, et al. Impact of potentially inappropriate prescribing on adverse drug events, health related quality of life and emergency hospital attendance in older people attending general practice: a prospective cohort study. J Gerontol A Biol Sci Med Sci 2017;72:271-7.

18. Cahir C, Bennett K, Teljeur C, et al. Potentially inappropriate prescribing and adverse health outcomes in community dwelling older patients. Br J Clin Pharmacol 2014;77:201-10.

19. Kelly A, Teljeur C. The national deprivation index for health \& health services research. Small Area Health Research Unit Technical Report: Small Area Health Research Unit, Trinity College Dublin, 2007.

20. World Health Organization. WHO collaborating centre for drug statistics methodology. ATC/DDD index 2011. World Health Organization2011WHO Collaborating Centre for Drug Statistics Methodology. ATC/DDD index. 2011.

21. National Guideline Centre UK. Multimorbidity: assessment, prioritisation and management of care for people with commonly occurring multimorbidity, 2016.

22. Barnett K, Mercer SW, Norbury M, et al. Epidemiology of multimorbidity and implications for health care, research, and medical education: a cross-sectional study. Lancet 2012;380:37-43.

23. Devlin N, Parkin D, Browne J. Using the EQ-5D as a performance measurement tool in the NHS. 2009.

24. Zigmond AS, Snaith RP. The hospital anxiety and depression scale. Acta Psychiatr Scand 1983;67:361-70.

25. Saliba D, Elliott M, Rubenstein LZ, et al. The vulnerable elders survey: a tool for identifying vulnerable older people in the community. J Am Geriatr Soc 2001;49:1691-9.

26. Higgins JP, Green S. Cochrane handbook for systematic reviews of interventions. John Wiley \& Sons. 2011;4

27. Chamnan $\mathrm{P}$, Simmons RK, Hori $\mathrm{H}$, et al. A simple risk score using routine data for predicting cardiovascular disease in primary care. $\mathrm{Br}$ $J$ Gen Pract 2010;60:e327-34

28. Smith SM, Ferede A, O'Dowd T. Multimorbidity in younger deprived patients: an exploratory study of research and service implications in general practice. BMC Fam Pract 2008;9:6

29. Yarnall AJ, Sayer AA, Clegg A, et al. New horizons in multimorbidity in older adults. Age Ageing 2017;46:882-8.

30. Perkins AJ, Kroenke K, Unützer J, et al. Common comorbidity scales were similar in their ability to predict health care costs and mortality. $J$ Clin Epidemiol 2004;57:1040-8.

31. Quail JM, Lix LM, Osman BA, et al. Comparing comorbidity measures for predicting mortality and hospitalization in three population-based cohorts. BMC Health Serv Res 2011;11:146.

32. Schneeweiss S, Wang PS, Avorn J, et al. Consistency of performance ranking of comorbidity adjustment scores in Canadian and U.S. utilization data. J Gen Intern Med 2004;19:444-50.

33. Brilleman SL, Salisbury C. Comparing measures of multimorbidity to predict outcomes in primary care: a cross sectional study. Fam Pract 2013;30:172-8

34. Wallace E, McDowell R, Bennett K, et al. Comparison of count-based multimorbidity measures in predicting emergency admission and functional decline in older community-dwelling adults: a prospective cohort study. BMJ Open 2016;6:e013089.

35. Boeckxstaens P, Vaes B, Van Pottelbergh G, et al. Multimorbidity measures were poor predictors of adverse events in patients aged $\geq 80$ years: a prospective cohort study. J Clin Epidemiol 2015;68:220-7

36. Boeckxstaens $P$, De Sutter A, Vaes B, et al. Should we keep on measuring multimorbidity? J Clin Epidemiol 2016;71:113-4. 